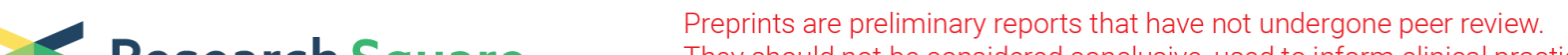 $\begin{array}{ll}\text { Research Square } & \begin{array}{l}\text { They should not be considered conclusive, used to inform clinical practice, } \\ \text { or referenced by the media as validated information. }\end{array}\end{array}$
}

\section{HPV prevalence and genotype distribution among women from Hengyang district of Hunan province, China}

\section{Shuang-yang Tang}

University of South China https://orcid.org/0000-0003-4692-4340

\section{S Ding}

University of South China

L Yu

University of South China

SY Shen

University of South China

YP Wan ( $\nabla$ muxin1022@126.com )

University of South China

YM Wu (D 57530760@qq.com )

University of South China

\section{Research article}

Keywords: human papillomavirus; prevalence; genotype; cervical cancer

Posted Date: May 5th, 2020

DOI: https://doi.org/10.21203/rs.3.rs-22870/v1

License: () (1) This work is licensed under a Creative Commons Attribution 4.0 International License.

Read Full License

Version of Record: A version of this preprint was published at Frontiers in Public Health on November 5th, 2021. See the published version at https://doi.org/10.3389/fpubh.2021.710209. 


\section{Abstract}

Background Human papillomavirus (HPV) infection can cause a variety of epithelial lesions, including even various malignant cancers, such as cervical cancer, vulvar cancer and etc. Most cervical cancers were closely associated with HPV infections. Therefore, understanding the ecological diversity of HPV prevalence and genotype distribution among various populations in different geographical regions was essential for optimizing HPV vaccination and maximizing vaccination effects. Aim This study was designed to investigate HPV infection prevalence and genotype distribution among women from Hengyang district of Hunan province in China.

Methods A total of 12053 patient data from the three-level hospitals in Hengyang city between July 2016 and July 2017 were retrospectively analyzed. The HPV positive was identified by Polymerase chain reaction (PCR). The HPV PCR-Flow fluorescence assay was used for HPV genotyping. The Chi-square test was performed to compare the differences among HPV infection rates or HR-HPV ratio-in-positives.

Results In this study, the HPV prevalence was $10.16 \%$ overall, and the multiple-type infection rate was $1.83 \%$. The HR-HPV infection rate was $8.52 \%$. The top six HPV genotypes were as follows in a descending order: HPV16, HPV58, HPV52, HPV39, HPV51, and HPV53. The patients who were above 60 years and below 21 years old were the most and second HPV prevalent group, respectively, the corresponding HRHPV infection rates were the most and the third, respectively. The infection rates of HPV and HR-HPV among outpatients were lower than those among hospitalized-patients, respectively. Among the hospitalized-patients, the infection rates of HPV and HR-HPV among 50 60 years group were both the most, following by those among above 60 years group. The HR-HPV ratio-in-positive among HPV-positive patients with histopathologic examination was higher than that among those patients without. Among 52 HPV-positive patients with cervical squamous carcinoma, the ratio-in-positive of HPV16 was $61.54 \%$.

Conclusions This study demonstrated that the HPV prevalence varied with the age among women from Hengyang district of Hunan province in China and showed their HPV genotype distribution that HPV16, HPV58, HPV52, HPV39, HPV51 and HPV53 infection were more popular in this region, which could provide the experimental basis for Chinese public health measures on cervical cancer prevention.

\section{Introduction}

Human papillomavirus (HPV) is a spherically non-enveloped and double-stranded DNA virus and prefers to infect the epithelial cells of skin and mucous membrane. HPV has more than 170 genotypes and its infection is widespread in the crowd [1]. According to the disease risk caused by HPVs, they were divided to low-risk HPV (LR-HPV) genotype and high-risk HPV (HR-HPV) genotype, among which those with sufficient or limited evidence in humans and strong mechanistic evidence for carcinogenicity were classified to HR-HPVs, and the others with inadequate epidemiological evidence and absence of carcinogenic potential in mechanistic studies were LR-HPVs. The HR-HPV types were those found most frequently (HPV 16, 18, 31, 33, 35, 45, 52, 58) and less constantly (HPV-39, 51, 56, 59) in cervical cancer, 
or classified as "probably carcinogenic to humans" (HPV 68) or "possibly carcinogenic" (HPV 26, 53, 66, $67,70,73,82,30,34,69,85,97)$ [2]. The clinical manifestations of HPV infection are various, which can be asymptomatic, benign warts, dysplasia, intractable pathological damages with high recurrence rate, and even can be converted into invasive cancer. Nearly all cervical cancer as well as a large proportion of epithelial cancers including some vulvar, vaginal, anal, penile and oropharyngeal cancers were caused by HPVs. The cervical cancer had become the second leading cause of women death globally, especially in developing country according to the reports of the International Agency for Cancer Research $[3,4,5]$. In China, the new case number of cervical cancer was 78400 in 2010 and mounted to 102000 in 2014, and that of cervical cancer deaths became 20000 in 2010 and 30400 in 2014 [6,7,8], suggesting cervical cancer had put a heavy burden on Chinese women, where HPV-related infections were the primary cause for their morbidity and mortality [4]. It was reported that due to the nation, socioeconomic status, age, ethnicity, or geographic location of populations was different, the HPV prevalence was substantially varied with different populations [9,10], and the HPV genotype distribution also differed in various regions worldwide. Hengyang city has 7.337 million of permanent residents from over 40 nationalities and is the deputy center of Hunan province in China, which also is the second-largest city in this province. Therefore, the HPV prevalence and HPV genotype distribution of this city is of significant for Chinese epidemiological data.

At present, the potentially long-term solution for preventing cervical cancer in developing countries is vaccination against HPV. The bivalent HPV vaccine (Cervarix) against HPV16/18 from GSK was firstly introduced to China in 2017 and the tetravalent vaccine (Gardasil) against HPV6/11/16/18 from Merck appeared in Chinese market in 2018, which can be possible to prevent most cervical cancers related their corresponding HPV genotype, respectively. Furthermore, the 9-valent HPV vaccine (2nd Gardasil) from Merck for HPV 6/11/16/18/31/33/45/52/58 was with broader coverage for cervical cancer and just had finished its conducting clinical trials in Chinese mainland during the working time of this paper. However, these vaccines were not only expensive but also scarce to Chinese women, even resulting in that the women with enough money had to wait the lucky lottery for vaccination. And due to that these HPV vaccines were not therapeutic but prophylactic to cervical cancer and had dissatisfied effects on married women, meanwhile which were introduced to Chinese women lately, their long-term effects was not clear on Chinese women and it even maybe fit them not very well, so the HPV infection of Chinese women should not be neglected. Therefore, the study on therapeutic vaccine or targeted-vaccine is still the hot spot for the cervical cancer prevention in China.

Prevention and control measures for HPV infections should be implemented according to their local situation [8]. Understanding the ecological diversity of HPV prevalence and its genotype distribution among various populations in different geographical regions of China is essential to optimize HPV vaccination and maximize its effects for Chinese women. Therefore, the infection prevalence and genotype distribution of HPV among women from Hengyang district of Hunan province in China were investigated in the current study so as to provide an important advice for Chinese public health measures on cervical cancer prevention. 


\section{Material And Methods}

\section{Data sources}

In this study, the data of 12053 women from the tertiary hospitals in Hengyang city between July 2017 and July 2018 were retrospectively analyzed. This retrospective analysis was in line with the Helsinki Declaration and approved by the ethical review committees of University of South China. No international review board (IRB) approval was required. The patients were from the gynecology department of hospital and with the suspected gynecologic disease. HPV testing was used as routine examination for those suspected gynecologic patients from better-off families. On the base of a series of medical tests, those outpatients with the suspected critical disease, such as the intraepithelial neoplasia or tumor in cervix, uterus, pelvic cavity and etc, would be transferred to in hospital for further examination and treatment.

\section{HPV genotyping}

The cervical samples were collected by the qualified gynecologists using a cyto-brush and stored in sampling tube with special cell preservation solution at $4{ }^{\circ} \mathrm{C}$ until testing HPV positive within a week. HPV positive was identified by Polymerase chain reaction (PCR), and HPV PCR-Flow fluorescence assay was used for HPV genotyping by HPV 27 genotyping KIT (Tellgen, Shanghai, China). HPV PCR-Flow fluorescence assay was conducted to amplify $27 \mathrm{HPV}$ genotypes, including $17 \mathrm{HR}-\mathrm{HPV}$ genotypes (HPV

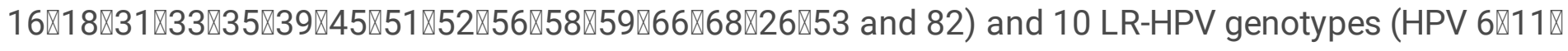
$40 \otimes 42 \rrbracket 43 \rrbracket 44 \rrbracket 55 \rrbracket 61 \rrbracket 81$ and 83$)$.

Pathological diagnosis All the pathologic tissue specimens were collected by two qualified gynecologists and stored as paraffin-embedded sections. The pathological diagnosis results were drawn by two experienced pathologists through hematoxylin-eosin (HE) stain Assay. The dysplastic changes beginning at the squamocolumnar junction in the uterine cervix that may be precursors of squamous cell carcinoma: cervical intraepithelial neoplasia (CIN) 1, mild dysplasia involving the lower one third or less of epithelial thickness; CIN2, moderate dysplasia with one third to two thirds involvement; CIN3, severe dysplasia or carcinoma in situ, with two thirds to full-thickness involvement.

\section{Statistical analysis}

The prevalence of HPV infection, presence of single or multiple HPV infections and HPV genotypes, as well as their corresponding $95 \%$ confidence intervals (Cls) were estimated with binomial distribution analysis. The Chi-square test was performed to compare the differences in the infection rates (the positive amount occupied among its corresponding whole population) of HPV or HR-HPV】and the ratioin-positive (the positive amount occupied among its corresponding positive population) of HR-HPV or LRHPV among various patients. A $p$-value of 0.05 was considered significant statistically. The statistical data analysis was performed by SPSS version 18.0 (SPSS, IBM, USA).

\section{Results}




\section{HPV prevalence among 12053 women}

A total of 1224 women were positive for HPV infection in 12053 women, among which 1003 were positive for a single genotype infection and 221 for multiple infections, so HPV infection rate was about $10.16 \%$ (1224/12053) overall, and the multiple-genotype infection rate was $1.83 \%$ (221/12053). Among the multiple genotype infections, a total of 163 patients were with double infections, 36 patients with treble infections and 12 patients with quadruple infections, and there were patients even with septuple or sextuple infections (Fig.1).

\section{Genotype distribution among $1224 \mathrm{HPV}$ positive women}

From 12053 patients, 27 different HPV genotypes were identified. Shown in Fig.2, 1027 women were infected by at least one type of HR-HPV, that is, HR-HPV infection rate was $8.52 \%$ (1027/12053). Among 12053 women, HPV16 was the most frequent genotype with the infection rate of $2.19 \%(264 / 12053)$. Moreover, the HPV16 positive patients occupied $21.57 \%$ among 1224 HPV positive patients, that is, the ratio-in-positive of HPV16 was $21.57 \%$ (264/1224). The distribution of remaining top five genotypes was as follows in a descending order: HPV58, HPV52, HPV39, HPV51 and HPV53. The amount of all clinical HPV strains found from 1224 positive women was 1538, among which 535 clinical HPV strains were from 221 women with multiple infections. Shown in Fig.2B, HR-HPVs occupied 82.57\% (1270/1538) and were more popular than LR-HPVs $(17.42 \%, 268 / 1538)$. Among the multiple infection genotypes, HPV16 was detected most frequently and HR-HPVs were more popular than LR-HPVs too (Fig.2C).

\section{Group distribution among 12053 patients}

Although the proportion of outpatients was $66.42 \%$ (8005/12053) more than that $(33.58 \%, 4048 / 12053)$ of hospitalized-patients, their HPV infection rate $(8.96 \%, 717 / 8005)$ was lower than that $(12.52 \%$, $507 / 4048)$ of hospitalized-patients $(p<0.01)$ (Fig.3A), the same as their multiple HPV infection rate $(1.17 \%, 94 / 8005 ; 3.14 \%, 127 / 4048)$ (Fig.3B). The difference of multiple-infection distribution between the outpatients and hospitalized-patients was not statistically significant ( $p>0.05)$ (Fig.3C).

\section{Age distribution of HPV prevalence}

Shown in Table 1, the ages of 12053 patients were focused on 21 to 50 years, which occupied $93.19 \%$ (11232/12053) and HPV-positive patients from 21 to 50 years correspondingly occupied $85.13 \%$ (1042/1224). Furthermore, the HPV infection rates of patients from above 60 years old and below 21 years old were $26.32 \%(50 / 190)$ or $23.21 \%(13 / 56)$, respectively, which were the most or second high $(p<0.01)$, respectively, and followed by that $(20.70 \%, 119 / 575)$ of $50 \sim 60$ years group.

Among all HPV infections, HR-HPV infection was identified as the infection with at least one HR-HPV genetype and its infection rate was $8.52 \%$ (1027/12053). The group of above 60 years old had the highest infection rate of both HPV $(26.32 \%, 50 / 190)$ and HR-HPV $(22.63 \%, 43 / 190)(p<0.01)$, on the contrary, the $50 \sim 60$ years group had the third most infection rate in HPV $(20.70 \%, 119 / 575)$ but the lowest in HR-HPV $(17.39 \%, 100 / 575)(p<0.01)$. Therefore, the ratio-in-positive of HR-HPV $(83.91 \%$, 
1027/1224) was higher than that of LR-HPV (16.09\%, 197/1224) among 1224 HPV-positive patients. Although HR-HPV ratio-in-positive of Group above 60 years old (86\%, 43/50) and below 20 years group $(69.23 \%, 9 / 13)$ were the most or least, respectively, the difference between them $(p>0.05)$ or among those of all age groups ( $p>0.05)$ had no statistical significance (Fig.4).

Table 1. HPV prevalence of different age groups among 12053 women

\begin{tabular}{ccccc}
\hline Age groups & \multicolumn{2}{l}{ HPV positive } & HPV negative & total \\
\cline { 2 - 3 } & HR & LR & & \\
\hline below 21 & 9 & 4 & 43 & 56 \\
\hline $21 \sim 30$ & 390 & 77 & 4679 & 5146 \\
\hline $31 \sim 40$ & 243 & 49 & 3221 & 3513 \\
\hline $41 \sim 50$ & 242 & 41 & 2290 & 2573 \\
\hline $51 \sim 60$ & 100 & 19 & 456 & 575 \\
\hline above 60 & 43 & 7 & 140 & 190 \\
\hline total & 1027 & 197 & 10829 & 12053 \\
\hline
\end{tabular}

Table 2. HPV prevalence of different age groups among 4048 hospitalized-patients

\begin{tabular}{ccccc}
\hline Age groups & \multicolumn{2}{c}{ HPV positive } & HPV negative & total \\
\cline { 2 - 3 } & HR & LR & & \\
\hline below 21 & 4 & 1 & 16 & 21 \\
\hline $21 \sim 30$ & 137 & 29 & 1690 & 1856 \\
\hline $31 \sim 40$ & 94 & 21 & 1075 & 1190 \\
\hline $41 \sim 50$ & 116 & 21 & 615 & 752 \\
\hline $51 \sim 60$ & 51 & 12 & 87 & 150 \\
\hline above 60 & 19 & 2 & 58 & 79 \\
\hline total & 421 & 86 & 3541 & 4048 \\
\hline
\end{tabular}

\section{HPV prevalence among hospitalized-patients}

Seen from Table 2, among 4048 hospitalized-patients, 507 patients was detected with HPV infections, among which 421 patients were infected with HR-HPVs and the other with only LR-HPVs. In the hospitalized-patients, the infection rate $(10.40 \%, 421 / 4048)$ and ratio-in-positive $(83.04 \%, 421 / 507)$ of HR-HPV were more high than those of only LR-HPV infection $(2.12 \%, 86 / 4048 ; 16.96 \%, 86 / 507)$, respectively. Although the difference of HR-HPV ratio-in-positive between the hospitalized-patients $(83.04 \%, 421 / 507)$ and outpatients $(84.52 \%, 606 / 717)$ was not significant statistically $(p>0.05)$, the HRHPV infection rate $(10.40 \%, 421 / 4048)$ of the hospitalized-patients was higher than that of the outpatients $(7.57 \%, 606 / 8005)(p<0.01)(p<0.01)$ and even the average level $(8.52 \%, 1027 / 12053)$. The investigated population were focused on 20 to 50 years old $(93.19 \%, 11232 / 12053)$ هso the same as the hospitalized-patients $(93.82 \%, 3798 / 4048)$. Moreover, the patients of this age bracket were the most $(82.45 \%, 418 / 507)$ among 507 HPV-infected hospitalized-patients. Among 4048 hospitalized-patients, the HPV infection rate $(42 \%, 63 / 150)$ and HR-HPV infection rate $(34 \%, 51 / 150)$ of $50 \sim 60$ years patients were both the most $(p<0.01, p<0.01)$, following by those of above 60 years patients $(26.58 \%, 21 / 79 ; 24.05 \%$, 19/79). Among 4048 hospitalized-patients, although the patients of above 60 years old had the highest HR-HPV ratio-in-positive $(90.48 \%, 19 / 21)$ and those below 21 years had the least $(80 \%, 4 / 5)$, they had on 
obvious difference in HR-HPV ratio-in-positive $(p>0.05)$, and that difference among all age groups had no statistical significance $(p>0.05)$ too (Fig.5).

\section{Pathomorphism distribution among 507 HPV-infected hospitalized-patients}

Among 507 hospitalized-patients with HPV infection, 284 patients had been performed with histopathologic examination according to their illness, but the other 223 patients did not need to be done. Among 284 HPV-infected hospitalized-patients with histopathologic examination, 70 patients were with cervical carcinoma or its precancerous lesions, 157 with uterine fibroid or its related lesions and 57 with pelvic neoplasm or its related lesions. Among 507 HPV-infected hospitalized-patients, the HR-HPV ratioin-positive of 284 patients with histopathologic examination was $90.49 \%(257 / 284)$ and higher than that $(78.03 \%, 174 / 223)$ of 223 patients without $(p<0.01)$ or even the average level $(83.04 \%, 421 / 507)(p<0.05)$. Among $284 \mathrm{HPV}$-infected hospitalized-patients with histopathologic examination, the patients with cervical carcinoma or its precancerous lesions had the highest HR-HPV ratio-in-positive $(95.71 \%, 67 / 70)$ $(p<0.05)$ (Fig.6).

Shown in Fig.6, among 70 HPV infected patients with cervical lesions or carcinoma, HR-HPV infection occupied $98.57 \%$ (69/70). Moreover, 52 patients were with cervical squamous carcinoma and HPV16 infections occupied $61.54 \%$ (32/52) therein. Among above 52 patients, the other top six genotypes were HPV52, HPV39, HPV51, HPV58, HPV18 and HPV53 (Fig.7), which was mainly consistent with the frequency of HPV16, HPV58, HPV52, HPV39, HPV51 and HPV53 infection in the whole population. It was worth mentioning that there was one patient with HPV6 single infection among cervical carcinoma patients.

\section{Discussion}

It was reported that HPV prevalence in worldwide women had significantly regional variation, for example in 2012 Africa had the highest HPV prevalence (24\%), which followed by Eastern Europe (21\%), Latin American (16\%) and South Eastern Asia (14\%) in turn [11]. In the current study, the overall HPV prevalence (10.16\%) in Hengyang district of Hunan Province was comparable to those reported in Zhejiang Province (13.3\%) [12] in China, and those reported in other countries, such as in Guatemala (13\%) [13] and in Iran, its Neighbouring Countries and Persian Gulf Area (0.62\% 25\%) [14], but was lower than those reported in some regions of China, such as Shenzhen City (15.9\%) [15], the northwestern Yunnan Province (18.4\%) [16], southern Yunnan Province (14.7\%) [17], Yangcheng County (14.8\%) [18], urban Tianjin (14.71\%) [19], Shenyang City (16.8\%) [20], south Taiwan (19.3\%) [21], and those reported in other countries, such as Denmark, Norway, and Sweden in 2006 2008 (36.5\%) or in 2012 2013 (34.5\%) [22]. The different HPV prevalence from various investigated people may be due to that these populations were from different geographical regions and with different nation, ethnicity, lifestyle or urbanization.

The rate of multiple HPV genotype infections (1.83\%) was similar with that in Beijing (1.5\%) from 2014 to 2015 [23], but lower than those in Yunnan Province (4.2\%) [16,24]. The high multiple-infection rate maybe cause the diseases more complicated or illness more serious, which was accordance with the report that 
the multiple-infections could elevate the risk of cervical cancer [25]. Therefore, the routine screening for $\mathrm{HPV}$ is very necessary to women's health.

In this study, the HR-HPV infection rate $8.45 \%$ was also a little lower than that found among women of Sichuan province (12.6\%) [26], those among Han women from Mojiang county of Yunnan Province (12.6\%) [27] and among the rural and urban women of southern Yunnan Province in China (11.4\%) [17]; and was comparable to those reported from Beijing City (9.9\%) [28], Tibet City (7.0\%) [29] or Zhejiang Province (10.2\%) [12] in China. Moreover, the infection rate of HR-HPV was more than that of exclusive LR-HPV among the investigated population, which was the same as among some population from Yunnan Province [17, 16], Shanxi Province [18], Taiwan Province [21] in China and some Amerindians [30]. The HPV infection rate of hospitalized group (12.52\%) was more than that of outpatients (8.96\%), the same as their multiple-infection rate $(1.17 \% ; 3.14 \%)$, due to the fact of the hospitalized patients had severer or more complicated illness than outpatients, which may be explained by that HPV infection, especially multiple HPV infection would worsen some gynaecologic diseases or cause some severe diseases occurred, meanwhile suggesting that it was urgent to develop the therapeutic vaccine for HPV infection. Moreover, the high HR-HPV ratio-in-positive among the HPV-positive hospitalized patients with histopathologic examination confirmed that HR-HPV can make the disease severer and even result in organic damage. Furthermore, the HR-HPV infections were more popular than the infections by exclusively LR-HPV among Amerindians, meanwhile the cervical abnormalities was consistent with their high HPV infection prevalence [30]. Similarly, the patients with cervical carcinoma or its precancerous lesions had the highest HR-HPV ratio-in-positive among the HPV-positive patients with histopathologic examination, suggesting cervical carcinoma or its precancerous lesions had close relationship with HRHPV virus.

All patients were with different gynecologic diseases and varied different degrees. The age-specific prevalence curve did not well agree with that conducted in highly developed countries [31,32]. Among all investigated patients, the top two HPV prevalent group were patients of above 60 years old $(26.32 \%)$ and below 21 years old $(23.21 \%)$, whose HPV infection rates were slightly higher than those of the most (younger women $<30$ years, $22 \%$ ) and second prevalent group (older ones $\geq 60$ years, $15 \%$ ) in Guatemala [13], respectively. Owing to that all patients were focused on from 21 to 50 years old (93.19\%) and the same as the hospitalized-patients (93.82\%), the HPV-positive patients of 21 50 years old occupied the majority among hospitalized-patients (82.42\%). The HR-HPV infection rate $(10.40 \%)$ of hospitalizedpatients was higher than the average level $(8.52 \%)$ and that of the outpatients $(7.57 \%)$, which also showed that the diseases related with HR-HPV infection would be more severe than those infected by LRHPV only, and moreover indicated the importance of therapeutic vaccine.

The highest HR-HPV infection rate of above 60 years group (22.63\%) in this study was obviously higher than that (7.4\%) in Beijing from 2014 to 2015, which may be because that the investigated population in Hengyang was from gynecologic patients and different with the population received free screening in Beijing [23]. Among hospitalized-patients, the HPV and HR-HPV infection rate (42\%; 34\%) of 50 60 years group were both the most and followed by those of above 60 years group $(26.58 \% ; 24.05 \%)$. The high 
prevalence of HPV and HR-HPV among above 50 patients was accordance with the situation that the cervical cancers likely to happen in 50-to-70-years-old patients, and might be attributable to their decreased immunity for clearing HPV infections, suggesting that the older people were the high risk population for HPV.

The HPV infection rate of the youngest group (below 21 years) was high and different with that of Xishuang Banna [16], but in line with the data reported in previous studies [29], suggesting that the higher urbanization may make the young women exposed to HPV infection more easily, which to a large extent may be ascribed to the early sexual activity. Moreover, the high HPV prevalence among below 21 years women showed that the HPV infection incidence had younger trend and should not be neglected, indicating that the young people should be improved their HPV-related knowledge more deep during the prevention and intervention for cervical cancer [33].

In this report, HPV16 was the most prevalent genotype, followed by HPV58, HPV52, HPV39, HPV51 and HPV53, which differed from HPV prevalence in Guatemala where the top six genotypes were HPV16, HPV18, HPV39, HPV58 , HPV52 and HPV45 [13]. Furthermore, the result of HPV genotype distribution was also different with that HPV52 had the most frequency followed by HPV39 and HPV68 among the urban population of Yunnan Province and some previous reports in China or other Asian countries [34,35]. However, this result was mainly consistent with the investigation in Wuhan City of Hubei Province where the most common genotype was HPV 52 followed by HPV 16, 58, 39 and 51[36], and in agreement with the results in the rural population of Yunnan Province [17] and in Shanxi Province of China [37], where the relatively high prevalence of HPV16, HPV58 and HPV52 infections were reported, confirming that HPV58 and HPV52 were also important for HPV infections in Asia. Among the HPV infected patients, the ratio-inpositive of HPV16 was $21.57 \%$, and near to that $(22 \%)$ in Guatemala [13], indicating that HPV16 was the most frequent as the general in all continents of the world. However, it could be seen that a slightly inferior ratio-in-positive (19.12\%) was from HPV58, suggesting that HPV58 was the same major infectedgenotype as HPV16 in China. In Western country or South American continents such as in Guatemala[13], HPV18 occupied the second frequency, but in Asian country HPV58 and HPV52 were more prevalent than HPV18 $[38,39]$. The variation of genotype distribution in Asia may be due to the different geographic regions, the diverse gene-interactions and various immune levels of patients $[38,40]$. Therefore, the introduced HPV vaccine from the West is not fit Chinese women very well, suggesting the study on native therapeutic vaccine is still important for Chinese researchers.

Among HPV-positive patients with cervical squamous carcinoma, the top seven genotype were HPV16, HPV52, HPV39, HPV51, HPV58, HPV18 and HPV53 \which was similar with that in Beijing from 2014 to 2015 [23]. Among above patients, the HPV16 ratio-in-positive (61.54\%) was comparable to that in Beijing from 2014 to 2015 (58.54\%) [23], and was obviously more than that among esophageal carcinoma samples (45.24\%) [41]. The high ratio-in-positive of HPV16 infection among cervical cancer sample indicated that HPV16 virus had played a key role in the development of cervical squamous carcinoma. It was worth mentioning that in this current study there was one cervical carcinoma patient with HPV 6 
single infection, suggesting that the role of LR-HPV in cervical squamous carcinoma could not be neglected.

Previously we had studied the awareness and knowledge levels about HPV among Chinese Pre-college students [42]. In this study we demonstrated that HPV prevalence and its genotype distribution in Hengyang city of Hunan province in China were mainly accorded with the present situation of HPV prevalence in China. The controlled variations may be occurred due to the different population in different socioeconomic and ethic environments with different lifestyles and life-standards from the different geographical regions. This study indicated that the HPV prevention and control strategies need to be carried out urgently for Hengyang city of Hunan in China and meanwhile highlighted the importance of HPV53 and HPV39 in China, which can guide the further study for the next generation of HPV vaccines.

\section{Declarations}

\section{Ethics approval and consent to participate}

The human data in present study were collected in line with the Helsinki Declaration and was approved by the ethical review committees of University of South China.

\section{Consent for publication}

Not applicable.

\section{Competing interests}

The authors declare that they have no competing interests.

\section{Funding}

This study was supported by the Provincial Natural Science Foundation of Hunan Province (No.2018JJ3452), the Educational Committee of Hunan Province (No.18B269) and the National Natural Science Foundation of China (No.81402169).

\section{Authors' contributions}

SY T analyzed and interpreted the patient data and was a major contributor in writing the manuscript. S $D, L Y$ and HY S also analyzed and interpreted the patient data collaboratively. YP W and YM W performed the collection of data. All authors read and approved the final manuscript.

\section{Acknowledgments}

Acknowledge the grants of the Foundation of Hunan Provincial Key Laboratory for Special Pathogens Prevention and Control Foundation (No. 2014-5), Construct Program of the "Double First-Class discipline" of University of South China (2020SYL). 


\section{References}

[1] de Villiers EM. Cross-roads in the classification of papillomaviruses. Virology. 2013 ;445(1-2):2-10.

[2] Bouvard V, Baan R, Straif K, Grosse Y, Secretan B, El Ghissassi F, Benbrahim-Tallaa L, Guha N, Freeman C, Galichet L, Cogliano V; WHO International Agency for Research on Cancer Monograph Working Group. A review of human carcinogens-Part B: biological agents. Lancet Oncol. 2009, 10(4):3212.

[3] Baay MF, Kjetland EF, Ndhlovu PD, Deschoolmeester V, Mduluza T, Gomo E, Friis H, Midzi N, Gwanzura L, Mason PR, Vermorken JB, Gundersen SG. Human papillomavirus in a rural community in Zimbabwe: the impact of HIV co-infection on HPV genotype distribution. J Med Virol 2004, 73: 481-485.

[4] Ferlay J, Shin HR, Bray F, Forman D, Mathers C, Parkin DM. Estimates of worldwide burden of cancer in 2008: GLOBOCAN 2008. Int J Cancer 2010, 127: 2893-2917.

[5] Flores-Pulido JJ, Martínez-Correa M. Cervical cancer and human papillomavirus. A glance from a family medical viewpoint. Rev Med Inst Mex Seguro Soc. 2015, 53 Suppl 2:S162-164.

[6] Shi JF, Canfell K, Lew JB, Qiao YL. The burden of cervical cancer in China: synthesis of the evidence. Int J Cancer 2012, 130: 641-652.

[7] Zhao FH, Hu SY, Zhang SW, Chen WQ, Qiao YL. Cervical cancer mortality in 2004-2005 and changes during last 30 years in China. Zhonghua Yu Fang Yi Xue Za Zhi 2010, 44: 408-412

[8] Gu XY, Zheng RS, Sun KX, Zhang SW, Zeng HM, Zou XN, Chen WQ, He J. Incidence and mortality of cervical cancer in China, 2014. Zhonghua Zhong Liu Za Zhi. 2018, 40(4):241-246.

[9] Li J, Kang LN, Qiao YL. Review of the cervical cancer disease burden in mainland China. Asian Pac J Cancer Prev 2010, 12: 1149-1153.

[10] Nakagawa M, Spencer HJ, Coleman HN, Greenfield WW. Distribution of human papillomavirus (HPV) types and anti-HPV T-cell immune responses among different racial/ethnic groups in Central Arkansas. J Ark Med Soc 2013, 109: 160-163.

[11] Forman D, de Martel C, Lacey CJ, Soerjomataram I, Lortet-Tieulent J, Bruni L, et al. Global burden of human papillomavirus and related diseases. Vaccine 2012, 30 (Suppl 5): F12-F23.

[12] Ye J, Cheng X, Chen X, Ye F, Lu W, Xie X. Prevalence and risk profile of cervical Human papillomavirus infection in Zhejiang Province, southeast China: a population-based study. Virol J 2010, 7: 66.

[13] Lou H, Gharzouzi E, Guerra SP, Domgue JF, Sawitzke J, Villagran G, Garland L, Boland JF, Wagner S, Rosas H, Troxler J, McMillen H, Kessing B, Alvirez E, Castillo M, Morales H, Argueta V, Rosingh A, van Aerde-van Nunen FJHB, Lopez G, Pinedo HM, Schiffman M, Dean M, Orozco R. Low-cost HPV testing and 
the prevalence of cervical infection in asymptomatic populations in Guatemala. BMC Cancer. 2018, 18(1):562.

[14] Sohrabi A, Hajia M. Cervical Cancer and Genital Infections: Assessment of Performance and Validation in Human Papillomavirus Genotyping Assays in Iran, its Neighbouring Countries and Persian Gulf Area. Iran J Pathol. 2017, 12:35-44.

[15] Liu ZH, Lin W, Wang YY, Wu B, Yuan SX, Yao JL, Zhao XS, Chen B, Qiao YL, Zhao FH, Chen W, Hu SY. Risk stratification of type-specific human papillomavirus for cervical precancers: evidence from a crosssectional study in Shenzhen. Zhonghua Zhong Liu Za Zhi. 2018;40(10):757-763.

[16] Baloch Z, Yuan T, Wang B, Tai W, Feng Y, Liu Y, Li X, Feng Y, Liu L, Zhang AM, Wu X, Xia X. Ethnic and geographic variations in HPV prevalence and genotype distribution in North-Western Yunnan, China. $J$ Med Virol 2016, 88: 532-540.

[17] Baloch Z, Yuan T, Yindi S, Feng Y, Tai W, Liu Y, Liu L, Zhang A, Wang B, Wu X, Xia X. Prevalence of genital human papillomavirus among rural and urban populations in southern Yunnan province, China. Braz J Med Biol Res. 2016, 49(6): e5254.

[18] Dai M, Bao YP, Li N, Clifford GM, Vaccarella S, Snijders PJ, Huang RD, Sun LX, Meijer CJ, Qiao YL, Franceschi S. Human papillomavirus infection in Shanxi Province, People's Republic of China: a population-based study. Br J Cancer 2006, 95: 96-101.

[19] Chen X, Wallin KL, Duan M, Gharizadeh B, Zheng B, Qu P. Prevalence and genotype distribution of cervical human papillomavirus (HPV) among women in urban Tianjin, China. J Med Virol. 2015, 87(11):1966-1972..

[20] Li LK, Dai M, Clifford GM, Yao WQ, Arslan A, Li N, Shi JF, Snijders PJ, Meijer CJ, Qiao YL, Franceschi S. Human papillomavirus infection in Shenyang City, People's Republic of China: A population-based study. Br J Cancer 2006, 95: 1593-1597.

[21] Lin H, Ma YY, Moh JS, Ou YC, Shen SY, Changchien CC. High prevalence of genital human papillomavirus type 52 and 58 infection in women attending gynecologic practitioners in South Taiwan. Gynecol Oncol 2006, 101: 40-45.

[22] Dillner J, Nygård M, Munk C, Hortlund M, Hansen BT, Lagheden C, Liaw KL, Kjaer SK. Decline of HPV infections in Scandinavian cervical screening populations after introduction of HPV vaccination programs. Vaccine. 2018, pii: S0264-410X(18)30626-1.

[23] Shen J, Gao LL, Zhang Y, Han LL, Wang JD. Prevalence of high-risk HPV and its distribution in cervical precancerous lesions among 35-64 years old women who received cervical cancer screening in Beijing. Zhonghua Yu Fang Yi Xue Za Zhi 2018, 52(5):493-497. 
[24] Sun LL, Jin Q, Li H, Zhou XR, Song ZQ, Cheng XM, Tao T, Liang B, Xu L, Wang YR, Zhen Y, He JW, Shen K. Population-based study on the prevalence of and risk factors for human papillomavirus infection in Qujing of Yunnan province, Southwest China. Virol J 2012, 9:153.

[25] Herrero R, Castle PE, Schiffman M, Bratti MC, Hildesheim A, Morales J, Alfaro M, Sherman ME, Wacholder S, Chen S, Rodriguez AC, Burk RD. Epidemiologic profile of type-specific human papillomavirus infection and cervical neoplasia in Guanacaste, Costa Rica. J Infect Dis 2005, 191: 1796-1807.

[26] He L, He J. Distribution of high-risk HPV types among women in Sichuan province, China: a crosssectional study. BMC Infect Dis. 2019;19(1):390.

[27] Baloch Z, Yue L, Yuan T, Feng Y, Tai W, Liu Y, Wang B, Li X, Liu L, Zhang AM, Wu X, Xia X. Status of human papillomavirus infection in the ethnic population in Yunnan Province, China. Biomed Res Int 2015, 2015: 314815.

[28] Li C,Wu M,Wang J, Zhang S, Zhu L, Pan J, Zhang W. A population based study on the risks of cervical lesion and human papillomavirus infection among women in Beijing, People's Republic of China. Cancer Epidemiol Biomarkers Prev 2010, 19: 2655-2664.

[29] Jin Q, Shen K, Li H, Zhou XR, Huang HF, Leng JH, Zhang WH, Gong XM, Cheng XM, Suo L, Zhu YC, Lang JH, Lu CM, Wang P, Mo WX. Prevalence of human papillomavirus infection in women in Tibet Autonomous Region of China. Zhonghua Fu Chan Ke Za Zhi 2009, 44: 898-902.

[30] Vargas-Robles D, Magris M, Morales N, de Koning MNC, Rodríguez I, Nieves T, Godoy-Vitorino F, Sánchez GI, Alcaraz LD, Forney LJ, Pérez ME, García-Briceño L, van Doorn LJ, Domínguez-Bello MG.High Rate of Infection by Only Oncogenic Human Papillomavirus in Amerindians. mSphere. 2018, 3(3). pii: e00176-18.

[31] Peto J, Gilham C, Deacon J, Taylor C, Evans C, Binns W, Haywood M, Elanko N, Coleman D, Yule $\mathrm{R}$, Desai M. Cervical HPV infection and neoplasia in a large populationbased prospective study: the Manchester cohort. Br J Cancer 2004, 91: 942-953.

[32] Franceschi S, Herrero R, Clifford GM, Snijders PJ, Arslan A, Anh PT, Bosch FX, Ferreccio C, Hieu NT, Lazcano-Ponce E, Matos E, Molano M, Qiao YL, Rajkumar R, Ronco G, de Sanjosé S, Shin HR, Sukvirach S, Thomas JO, Meijer CJ, Muñoz N. Variations in the age-specific curves of human papillomavirus prevalence in women worldwide. Int J Cancer 2006, 119: 2677-2684.

[33] Preston SM, Darrow WW.Improving Human Papillomavirus-Related Knowledge and Attitudes Among Ethnically Diverse Young Adults. Health Equity. 2019, 3(1):254-263.

[34] Clifford GM, Gallus S, Herrero R, Munoz N, Snijders PJ, Vaccarella S, Anh PT, Ferreccio C, Hieu NT, Matos E, Molano M, Rajkumar R, Ronco G, de Sanjosé S, Shin HR, Sukvirach S, Thomas JO, Tunsakul S, Meijer CJ, Franceschi S; IARC HPV Prevalence Surveys Study Group. Worldwide distribution of human 
papillomavirus types in cytologically normal women in the International Agency for Research on Cancer HPV prevalence surveys: a pooled analysis. Lancet 2005, 366: 991-998.

[35] Yamasaki K, Miura K, Shimada T, Miura S, Abe S, Murakami M, Sameshima T, Fujishita A, Kotera $\mathrm{K}$, Kinoshita A, Yoshiura K, Masuzaki H. Epidemiology of human papillomavirus genotypes in pregnant Japanese women. J Hum Genet 2011, 56: 313-315.

[36] Xiang F, Guan Q, Liu X, Xiao H, Xia Q, Liu X, Sun H, Song X, Zhong Y, Yuan CH, Xiang Y. Distribution characteristics of different human papillomavirus genotypes in women in Wuhan, China. J Clin Lab Anal. 2018, e22581.

[37] Xu H, FH, Gao XH, Hu SY, Chen JF, Liu ZH, Xu XL, Gao LM, Liu QG, Ma L, Liu YJ, Qiao YL. Costeffectiveness analysis on the once-in-a-lifetime cervical cancer screening program for women living in rural and urban areas of China. Zhonghua Liu Xing Bing Xue Za Zhi 2013, 34: 399-403.

[38] de Sanjose S, Diaz M, Castellsague X, Clifford G, Bruni L, Munoz N, Bosch FX. Worldwide prevalence and genotype distribution of cervical human papillomavirus DNA in women with normal cytology: a metaanalysis. Lancet Infect Dis 2007, 7: 453-459.

[39] Bosch FX, Burchell AN, Schiffman M, Giuliano AR, de Sanjose S, Bruni L, Tortolero-Luna G, Kjaer SK, Muñoz N. Epidemiology and natural history of human papillomavirus infections and type-specific implications in cervical neoplasia. Vaccine 2008, 26 (Suppl 10): K1-16.

[40] Hildesheim A, Wang SS. Host and viral genetics and risk of cervical cancer: a review. Virus Res 2002, 89: 229-240.

[41] Li S, Shen H, Li J, Hou X, Zhang K, Li J. Prevalence of the integration status for human papillomavirus 16 in esophageal carcinoma samples. Turk J Gastroenterol. 2018, 29(2):157-163.

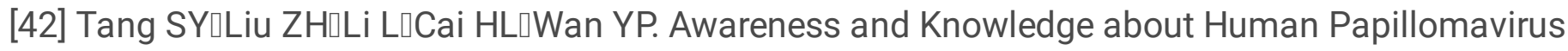
among High School Students in China. J Reprod Med 2014, 59(1-2):44-50.

\section{Figures}




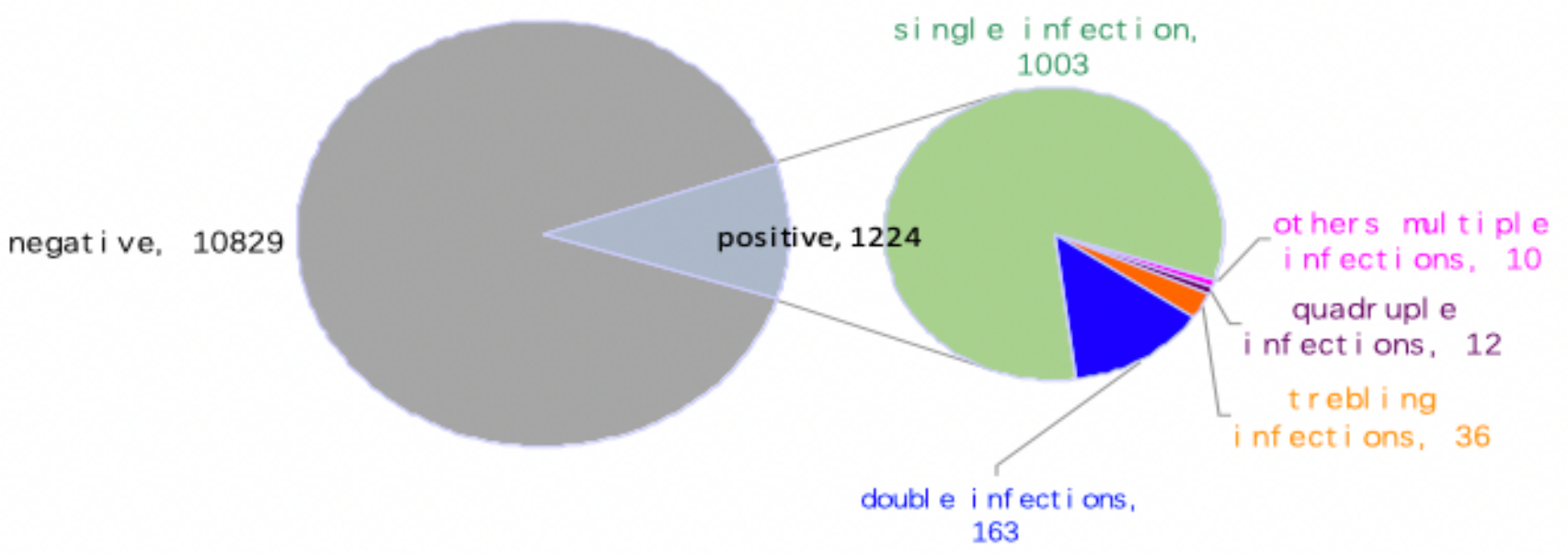

Figure 1

Human papillomavirus prevalence among 12053 women 


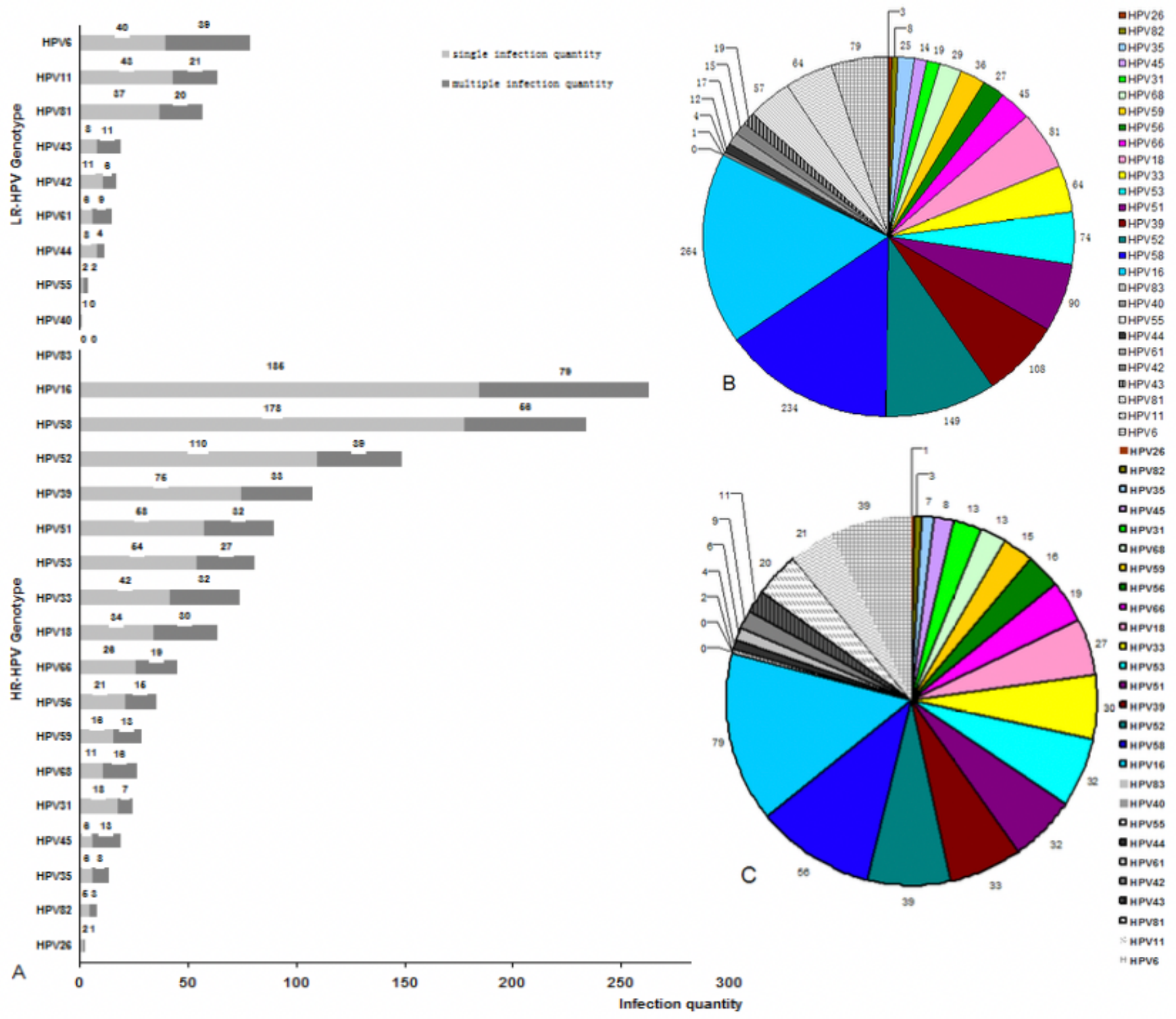

Figure 2

Genotype distribution among 1224 HPV positive women. A: Genotype distribution of 1538 clinical HPV strains B: Genotype proportion of 1538 clinical HPV strains C: Genotype proportion of 535 clinical HPV strains from multiple infections 

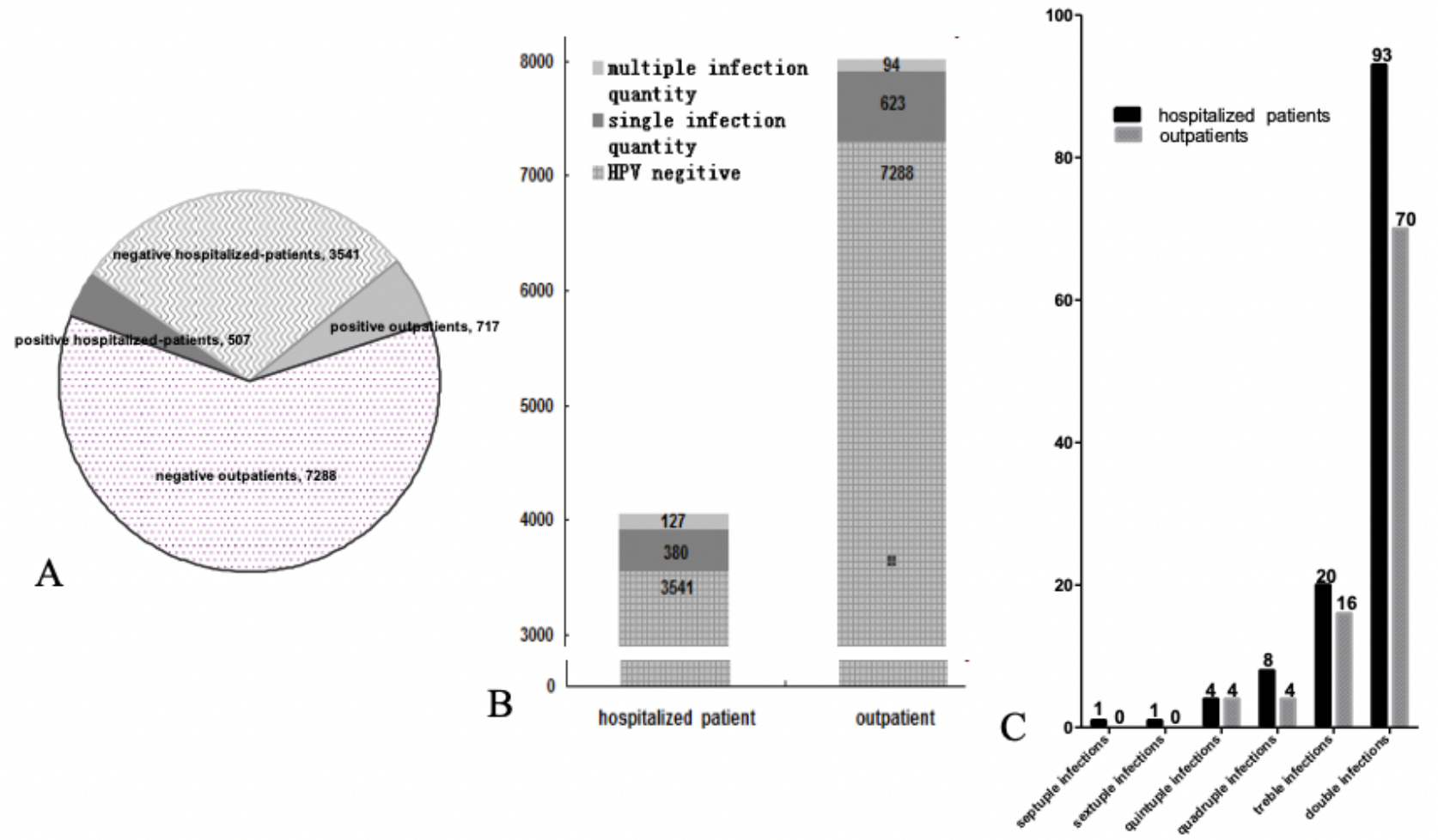

Figure 3

Group distribution among 12053 patients. A: Group distribution of HPV infection B: Group distribution of multiple HPV infection C: Distribution of multiple HPV infections between outpatients and hospitalizedpatients

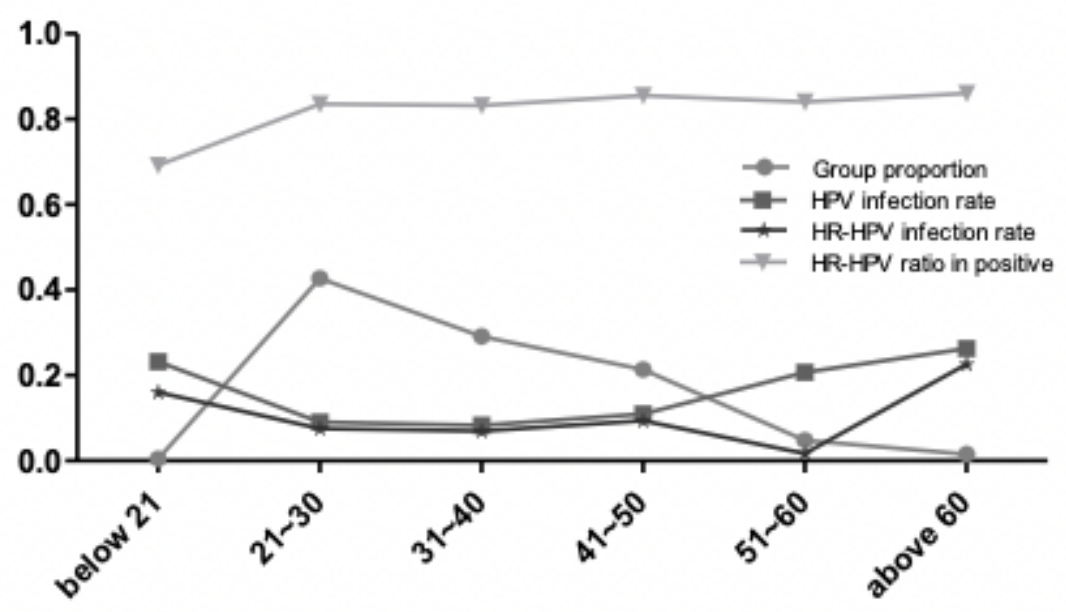

Figure 4

HPV prevalence of different age groups among 12053 women 


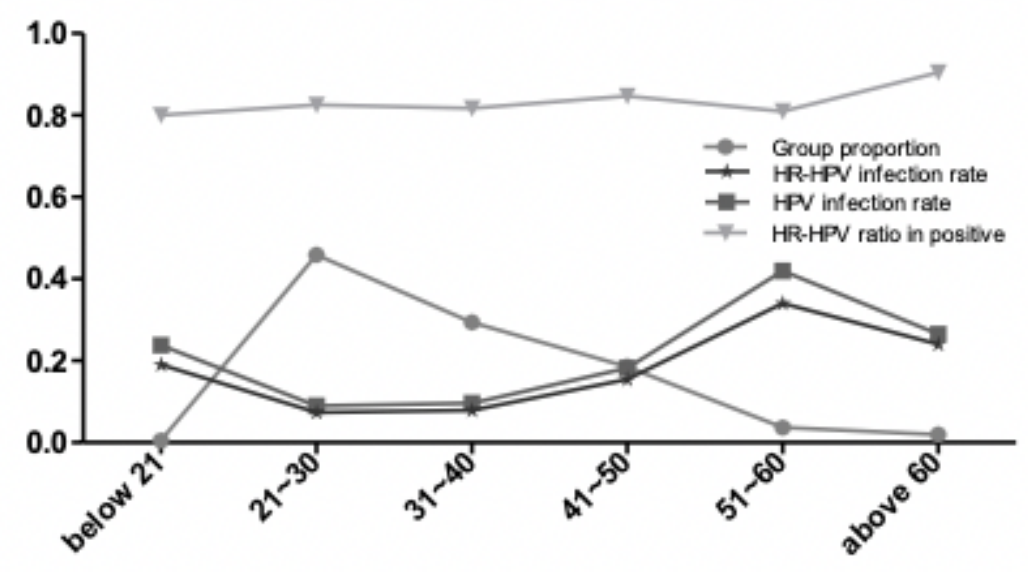

Figure 5

HPV prevalence of different age groups among 4048 hospitalized-patients

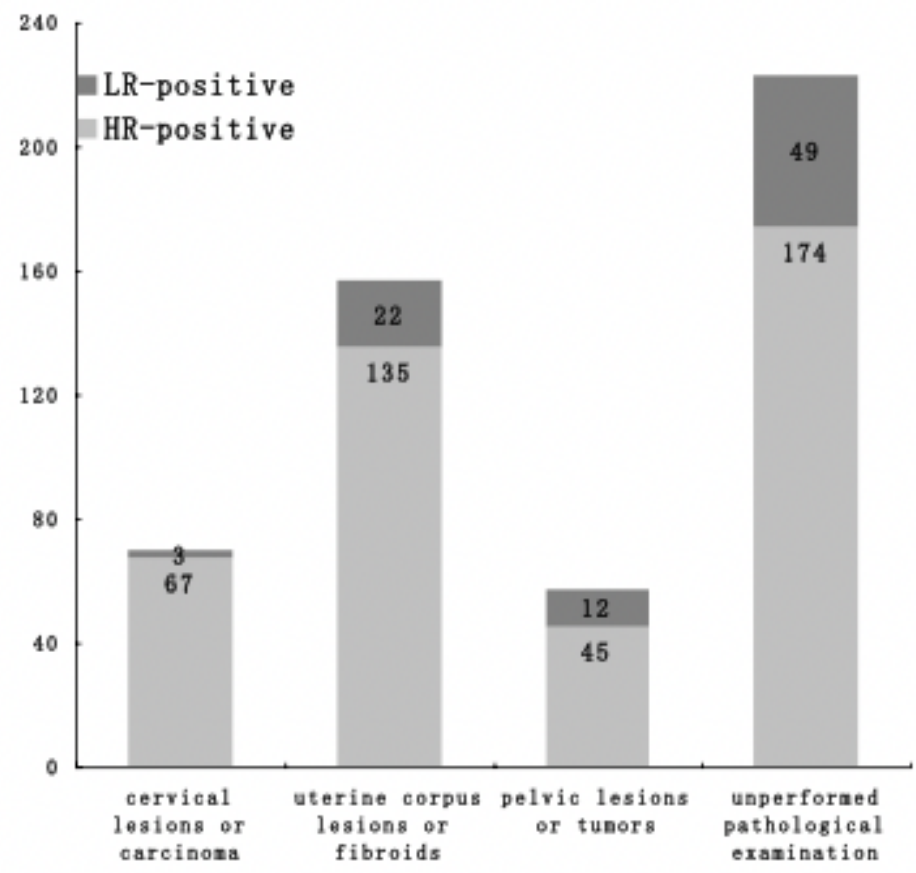

Figure 6

Pathomorphism distribution among 507 HPV positive 


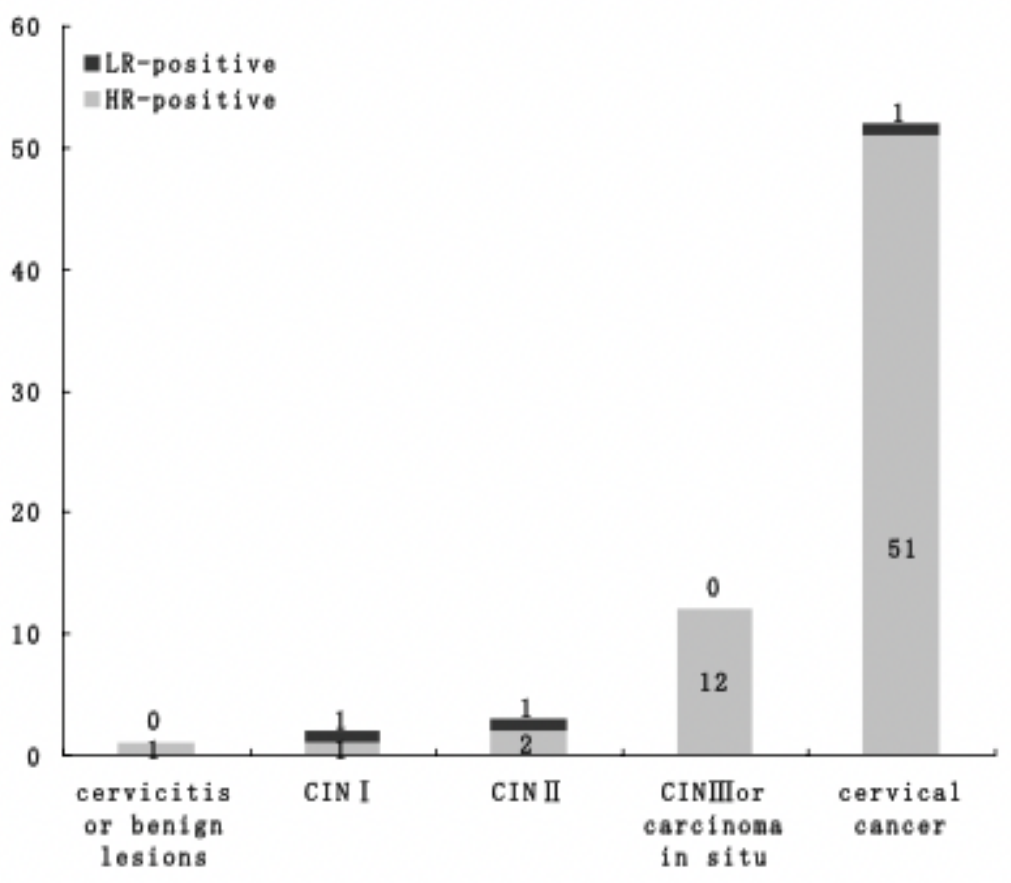

Figure 7

HPV prevalence among 70 HPV positive patients with cervical lesions or carcinoma carcinoma 